\title{
Melanoma of unknown primary origin coexisting with early-onset multifocal basal cell carcinoma
}

\author{
Magdalena Kiedrowicz¹, Mirosław Halczak², Józef Kładny², Andrzej Królicki' ${ }^{1}$, Romuald Maleszka ${ }^{1}$
}

${ }^{1}$ Department of Dermatology and Venereology, Pomeranian Medical University, Szczecin, Poland

Head of the Department: Prof. Romuald Maleszka MD, PhD

${ }^{2}$ Department of Oncological Surgery, Pomeranian Medical University, Szczecin, Poland

Head of the Department: Prof. Józef Kładny MD, PhD

Postep Derm Alergol 2015; XXXII (4): 320-322

DOI: $10.5114 /$ pdia.2014.40972

Skin is the most common localization of primary melanoma, however in 2-6\% of patients a metastasis of melanoma without a detectable primary tumor is found [1]. This is referred to as melanoma of unknown primary origin (MUP). It usually presents as cutaneous or subcutaneous nodules or lymph node metastases [2]. The theories concerning the etiology of MUP include the presence of a melanoma that was misdiagnosed and excised without the further histopathological examination, a regressed melanoma; or a primary nodal melanoma connected with malignant transformation of a nevus cell in a lymph node or other non-skin tissue. In support of the last two theories, in melanomas a partial spontaneous regression connected with immunological mechanisms frequently occurs, moreover ectopic melanocytes may be found in lymph nodes and the other tissues, being a potential reservoir of the cells, which may undergo a neoplastic transformation [2]. In patients with MUP metastases may be found primarily in the skin, subcutaneous tissue, internal organs, bones and brain [1]. In more than $50 \%$ of MUP cases, similarly to sporadic melanomas with a known primary origin, a mutation of the gene encoding kinase protein BRAF (V600E) is found. The coexistence of melanoma with the other skin cancers may be connected with the presence of genes encoding the factors regulating the cell cycle, e.g. p53 protein.

A female patient with metastasis of melanoma to the lymph node of the right groin of unknown primary origin, with a coexistence of three basal cell carcinomas is presented in the report.

A 33-year-old female patient, generally healthy, with a positive history of drug abuse in the past, presented with a few centimeters' large, painless nodule within the right inguinal region. Ultrasound examination revealed a longitudinal tumor, partly solid and partly polycystic with vascularized septa. The nodule was surrounded by the capsule and revealed no features of infiltration of the adjacent tissues (Figure 1). Histopathological examination of an excised lesion showed the presence of nodal tissue with the metastasis of melanoma with HMB45 positive, S-100 positive, Melan-A positive, V-9 positive, MIB-1 (a proliferation marker which recognizes the Ki-67 antigen) locally positive (Figures $2 \mathrm{~A}, \mathrm{~B}$ ). Keratin AE1/AE3, CG30 and leucocyte common antigen (LCA) were negative in neoplastic cells. There was no history of any surgically excised melanocytic or pigmented lesions. The patient declared extensive exposure to ultraviolet in the past, but without any episodes of sun burns. Family melanoma history was negative, however the patient's mother had a history of one basal cell carcinoma (BCC) at the age of 62 .

Lymphadenectomy of the whole group of lymph nodes within the right inguinal region revealed no metastases to the other nodes. Dermatological examination, performed after the diagnosis of MUP, revealed the presence of numerous solar lentigines and melanocytic nevi on the trunk and extremities with features of atypical lesions in macroscopic and dermoscopic evaluation in some of them. Moreover, the existence of two nodular lesions without dermoscopic features of melanocytic nevi were diagnosed on the abdomen, as well as a single erythematous patch in the left scapular area. Seventeen suspected skin lesions were qualified for surgical removal. The excised nevi revealed histopathological features of benign lesions, no melanoma cells were found in the biopsy specimens. Two nodular lesions on the abdomen and the macular one within the left scapular area showed histopathological features of basal cell carcinoma. Laboratory analyses were within the normal range. The activity of lactate dehydrogenase was within the normal range. A detailed diagnostic imaging, including whole-body positron emission tomography-computed tomography (PET-CT) screening, computed tomography of the abdomen, pelvis, chest and head, endoscopy of the

Address for correspondence: Magdalena Kiedrowicz, Department of Dermatology and Venereology, Pomeranian Medical University, 2 Siedlecka St, 72-010 Police, Poland, phone/fax: +48 9142539 24/+48 9142539 25, e-mail: magkied@poczta.onet.pl Received: 9.12.2013, accepted: 7.01.2014. 
gastrointestinal tract, ultrasound examination of breast and the female genital organ, gynecological and ophthalmic evaluation, examination of the anal region confirmed neither the presence of primary melanoma nor metastases in the other localizations. A mutation in codon V600 of the gene encoding BRAF kinase protein was confirmed in the patient. Further genetic evaluation (assessment of mutations in BRCA1 and CHEK2 genes) confirmed no features of high hereditary cancer susceptibility. Regarding the absence of primary origin of melanoma, differentiation between regional and distant metastases, as well as an evaluation of clinical staging (stage IIIb or IV according to AJCC/UICC staging system) and prognostic factors could not be performed. No indications for the further complementary adjuvant treatment were established.

The first exclusion criteria for diagnosis of a true unknown primary melanoma were defined by Das Gupta et al. in 1963 [3]. They included the history of orbital enucleation or exenteration, history of a skin lesion removed, the presence of a scar of previous local treatment in the area drained by the involved lymph node and lack of a thorough physical assessment, including ophthalmic, anal and genital examination.

Our patient confirmed the presence of some skin lesion on the right buttock that was mechanically removed by scratching a few years ago. Skin biopsy from the remaining slightly pigmented macule at this site revealed only a scant inflammatory infiltration with lymphoid cells. No other evidence of potential regression was found. The hypothesis of primary origin of melanoma in the lymph node was also considered. No evidence of primary tumor in thorough physical assessment could confirm this assumption in the presented case [4, 5]. Unfortunately, there are no clear criteria defining the diagnosis of this variant of melanoma. Moreover, the literature data lack certain descriptions with histological confirmation of primary nodal melanoma.

Another unusual observation was the coexistence of MUP with multifocal BBC in the presented female. Mel-

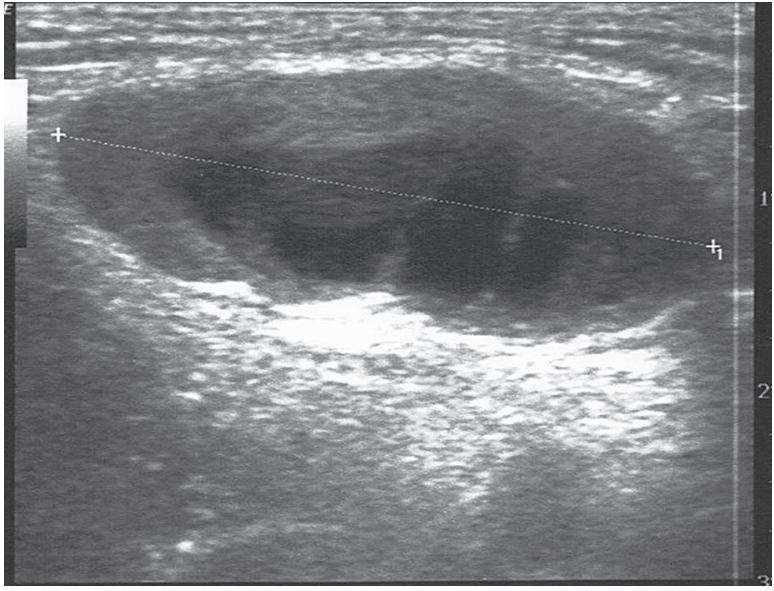

Figure 1. An ultrasound picture of an enlarged lymph node within the right inguinal region

anoma patients may develop a second malignancy. The most common are second skin tumors, with the highest incidence of BCCs and multiple melanomas [6]. In one study, second malignancies were seen in $11.0 \%$ of all melanoma patients, BCCs accounted for one-fourth of all cases. However, the mean age at the time of melanoma and $B B C$ diagnosis was over 60 years [6]. The incidence of BCC in the population younger than 40 years is low, however it is increasing, most likely due to the coexistence of multiple risk factors, with extensive exposure to UV as the most important one [7]. Also genetic defects, as in basal cell nevus syndrome, Muir-Torre syndrome and xeroderma pigmentosum are risk factors for the accelerated development of early-onset BCC. A decrease in DNA repair may determine the genetic susceptibility of non-melanoma skin cancer at a young age [7]. The presented patient had no noticeable symptoms of any genetic syndrome coexisting with a higher risk of BCC development. However, she declared a cumulative sun exposure in the past and that, with concomitance of
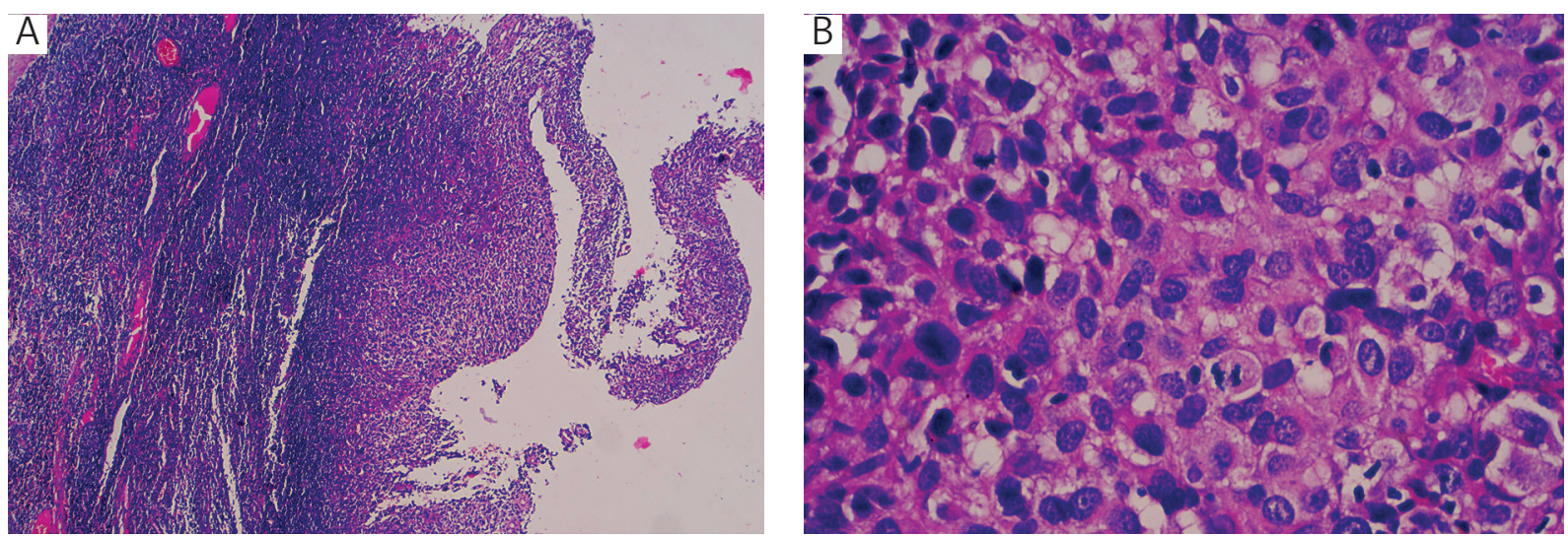

Figure 2. Histopathological examination of an excised tumor presenting nodal tissue with the metastasis of melanoma. A $-40 x$. B $-400 x$ 
defects in DNA repair, could have resulted in the development of melanoma and non-melanoma skin cancer. To the best our knowledge, this is the first description of MUP coexisting with early-onset multifocal BCC diagnosed at the same time.

\section{Conflict of interest}

The authors declare no conflict of interest.

\section{References}

1. Schlagenhauff B, Stroebel W, Ellwanger U, et al. Metastatic melanoma of unknown primary origin shows prognostic similarities to regional metastatic melanoma: recommendations for initial staging examinations. Cancer 1997; 80: 60-5.

2. O’Neill JK, Khundar R, Knowles L, et al. Melanoma with an unknown primary: a case series. J Plast Reconstr Aesthet Surg 2010; 63: 2071-80.

3. Das Gupta T, Bowden L, Berg JW. Malignant melanoma of unknown primary origin. Surg Gynecol Obstet 1963; 117: 341-5.

4. Kamińska-Winciorek G, Placek W. The most common mistakes on dermatoscopy of melanocytic lesions. Postep Derm Alergol 2015; 32: 33-9.

5. Kamińska-Winciorek G, Właszczuk P, Wydmiński J. "Mistletoe sign": probably a new dermoscopic descriptor for melanoma in situ and melanocytic junctional nevus in the inflammatory stage. Postep Derm Alergol 2013; 30: 316-9.

6. Wolff J, Wollina U. Second malignancies in melanoma patients in Thuringia. J Eur Acad Dermatol Venereol 2000; 14 479-83.

7. Christenson LJ, Borrowman TA, Vachon CM, et al. Incidence of basal cell and squamous cell carcinomas in a population younger than 40 years. JAMA 2005; 294: 681-90. 